\title{
A UNIFIED VIEW OF METHODS TO RESOLVE THE INVERSE PROBLEM OF TEXTURE GONIOMETRY
}

\author{
H. SCHAEBEN \\ Guerickestr. 62, 66123 Saarbrücken, FRG
}

(Received 31 August 1995)

\begin{abstract}
Invariants of the tomographic projection operator of texture goniometry and counterexamples provide a unified view of existing methods to resolve the corresponding inverse problem and may be instructive to develop novel approaches.
\end{abstract}

KEY WORDS: Spherical harmonics, $\delta$-distributions, indicators; Riemann-Lebesgue kernel, de la Vallée Poussin kernel, Abel-Poisson kernel (Cauchy distribution), Gauss-Weierstrass kernel (Brownian distribution); vou Mises-Fisher distribution, Bingham distribution.

\section{INTRODUCTION}

To analyse and interpret patterns of preferred crystal orientation, a probability density function $f$ defined on the group $S O(3)$ of rotations referred to as orientation density function has to be evaluated. The orientation density function cannot generally be measured without destroying the specimen of the material to be investigated. An experimentally accessible corresponding pole density function $\tilde{P}_{h}$ of a given crystal form $h$ is defined as superposition of tomographic projections of the orientation density function provided by an integral operator $P_{\mathrm{x}}: \mathcal{L}^{2}(S O(3)) \mapsto \mathcal{L}^{2}\left(S^{3}\right)$. Applying functions the type of which is preserved by the projection operator or which are otherwise wellbehaved provides means to resolve the corresponding inverse problem and may largely facilitate numerical evaluation in orientation imaging microscopy.

\section{BASIC MATHEMATICS OF TEXTURE GONIOMETRY}

Let $f \in \mathcal{L}^{2}(G)$ be a square integrable orientation density function (odf) defined on an appropriate subgroup $G$ of the group $S O(3)$ of proper rotations.

Let $\mathrm{x}, \mathrm{y} \in S^{3} \subset I R^{3}$, then the integral operator $\mathrm{P}_{\mathrm{x}}: \mathcal{L}^{2}(G) \mapsto \mathcal{L}^{2}\left(S^{3}\right)$ is defined as

$$
\left(\mathcal{P}_{\mathrm{x}} f\right)(\mathrm{y})=\mathcal{P}_{\mathrm{x}}[f(g)](y)=\frac{1}{2 \pi} \int_{\{g \in G \mid \mathrm{x}=g \mathrm{y}\}} f(g) d g=P_{\mathrm{x}}(\mathrm{y})
$$

Obviously, $P_{-\mathrm{x}}(\mathrm{y})=P_{\mathrm{x}}(-\mathrm{y})$ 
An odf $f$ is related to its corresponding pole density function (pdf) $\tilde{P}_{h}$ of the crystal form $h=\left\{\mathrm{h}_{m} \mid m=1, \ldots, M_{h}\right\}$ defined for $\mathrm{r} \in S^{3} \subset I R^{3}$ by

$$
\left(\tilde{P_{h}} f\right)(\mathrm{r})=\frac{1}{M_{h} / 2} \sum_{m=1}^{M_{h} / 2}\left(\frac{1}{2}\left[\mathrm{P}_{\mathrm{h}_{m}}+\mathrm{P}_{-\mathrm{h}_{m}}\right] f\right)(\mathrm{r})=\tilde{P}_{h}(\mathrm{r})
$$

with $\mathrm{h}_{m} \in S_{+}^{3}, m=1, \ldots, M_{h} / 2$, where $M_{h}$ denotes the multiplicity of the crystal form $h$.

Thus, a pdf is an even function

$$
\tilde{P}_{h}(\mathrm{r})=\tilde{P}_{h}(-\mathrm{r})
$$

while an odf is generally neither even nor odd.

\section{INVARIANTS OF THE PROJECTION OPERATOR}

\subsection{Harmonics}

As is well known since the early days of quantitative texture analysis, applying the pole figure projection operator (1) to generalized spherical harmonics

$$
\begin{aligned}
\mathrm{P}_{ \pm \mathrm{x}}\left[D_{m, n}^{l}\left(g^{-1}\right)\right](\mathrm{y}) & =\int_{\mathrm{G}} \delta_{3}(\mathrm{x}-g \mathrm{y}) D_{m, n}^{l}\left(g^{-1}\right) d g \\
& =( \pm 1)^{l} \frac{2 \pi}{2 l+1} Y_{l, m}^{*}(\mathrm{x}) Y_{l, n}(\mathrm{y})
\end{aligned}
$$

yields spherical harmonics (Bunge, 1969; 1982; Matthies et al., 1987). Since harmonics are either even or odd, depending on $l$, it holds that

$$
\begin{aligned}
\tilde{P}_{\mathrm{x}}\left[D_{m, n}^{l}\left(g^{-1}\right)\right](\mathrm{y}) & =\left(\mathrm{P}_{\mathrm{x}}+\mathrm{P}_{-\mathrm{x}}\right)\left[D_{m, n}^{l}\left(g^{-1}\right)\right](\mathrm{y}) \\
& = \begin{cases}\frac{4 \pi}{2 l+1} Y_{l, m}^{*}(\mathrm{x}) Y_{l, n}(\mathrm{y}) & \text { if } l \text { even } \\
0 & \text { if } l \text { odd }\end{cases}
\end{aligned}
$$

Spherical harmonics lead to orthonormal series expansion. It may be noted that classical harmonic series expansion was developed by 19 th century mathematicians as a means of representation and not as a means of numerical evaluation.

\section{$3.2 \delta$ - distributions}

Simply, the pole figure projection operator (1) maps a $\delta$-distribution onto another $\delta$-distribution

$$
\begin{aligned}
P_{\mathrm{x}}\left[\delta_{4}\left(g g_{0}^{-1}\right)\right](\mathrm{y}) & =\frac{1}{2 \pi} \int_{G} \delta_{3}(\mathrm{x}-g \mathrm{y}) \delta_{4}\left(g g_{0}^{-1}\right) d g \\
& =\frac{1}{2 \pi} \delta_{3}\left(\mathrm{x}-g_{0} \mathrm{y}\right)
\end{aligned}
$$

$\delta$-distributions provide the most simple model odf and pdf, respectively, which are defined pointwise. 


\subsection{Central functions, rotationally invariant functions}

Let $\omega=\omega\left(g g_{0}^{-1}\right) \in[0, \pi]$ denote the orientation distance of the orientations $g, g_{0} \in$ $G$, let $\eta=\eta\left(\mathrm{y}, g_{0}^{-1} \mathrm{x}\right)=\arccos \left(\mathrm{y} g_{0}^{-1} \mathrm{x}\right)$ denote the angle between $\mathrm{y}$ and $g_{0}^{-1} \mathrm{x}$; and let $D_{n}$ denote the Dirichlet kernel (cf. Butzer and Nessel, 1971)

$$
\begin{aligned}
D_{n}(\omega) & =\sum_{m=-l}^{l} D_{m, m}^{l}\left(g_{0}^{-1} g\right)=\sum_{m, n=-l}^{l} D_{m, n}^{l^{*}}\left(g_{0}^{-1}\right) D_{m, n}^{l}\left(g^{-1}\right) \\
& =1+2 \sum_{l=1}^{n} \cos (l \omega)=\left\{\begin{array}{ll}
\frac{\sin ((2 n+1) \omega / 2)}{\sin (\omega / 2)} & \omega \neq 2 j \pi \\
2 n+1 & \omega=2 j \pi
\end{array} \quad j \in \mathbb{Z}(7)\right.
\end{aligned}
$$

which may also be referred to as Chebyshev polynomial of second kind, first Gegenbauer ultraspherical polynomial, or Legendre polynomial for $I R^{4}$.

An orientation density function is called central, if it depends only on the orientation distance with respect to a given orientation $g_{0}$. In this case, the harmonic series expansion simplifies to

$$
f\left(g ; g_{0}, \kappa\right)=f\left(\omega\left(g_{0}^{-1} g\right) ; \kappa\right)=\sum_{l=0}^{\infty} C_{l}(\kappa) D_{l}(\omega)
$$

with coefficients

$$
C_{l}(\kappa)=\frac{2}{\pi} \int_{[0, \pi]} f\left(g ; g_{0}, \kappa\right) D_{l}(\omega) \sin ^{2}(\omega / 2) d \omega
$$

where $\kappa$ denotes a measure of spread, or concentration, respectively, with respect to $g_{0}$.

Applying the operator (1) to an orientation density function $f$ which is central with respect to an arbitrary $g_{0} \in G$

$$
P_{\mathrm{x}}\left[f\left(g ; g_{0}, \kappa\right)\right](\mathrm{y})=\sum_{l=0}^{\infty} C_{l}(\kappa) P_{l}\left(\mathrm{x} g_{0} \mathrm{y}\right)=P_{\mathrm{x}}\left(\mathrm{y} ; g_{0}^{-1} \mathrm{x}, \kappa\right)
$$

results in a function $P_{\mathrm{x}}$ which is rotationally invariant with respect to $y_{0}=g_{0}^{-1} \mathrm{x} \in$ $S^{3}$,cf. (Matthies et al., 1988). Thus, rotational invariance is preserved by the operator $P_{\mathrm{x}}$.

A pole density function corresponding to a given central orientation density function $f$ is the superposition of rotationally symmetric functions $P_{\mathrm{h} m}, m=1, \ldots, M_{h}$, where $M_{h}$ denotes the multiplicity of the crystal form $h$. Since $\mathrm{h}_{m} \in h \subset S^{3}$ implies $-\mathrm{h}_{m} \in h$, it can essentially be stated that a pole density function corresponding to a given central orientation density function $f$ is the superposition of even functions $\tilde{P}_{\mathrm{h} m}$ on $S_{+}^{3}$ centered at $\mathrm{r}_{m}= \pm g_{0}^{-1} \mathrm{~h}_{m} \in S_{+}^{3}, m=1, \ldots, M_{h} / 2$.

\subsection{De la Vallée Poussin kernel}

Applying the pole figure projection operator (1) to the de la Vallée Poussin kernel (cf. Butzer and Nessel, 1971)

$$
\mathcal{V}_{k}(\omega)=C v(k) \cos ^{2 k}(\omega / 2), \omega \in[0, \pi], k \in I N, k_{0}=\infty
$$

yields

$$
P_{\mathrm{x}}\left[\mathcal{V}_{k}(\omega)\right](\mathrm{y})=\left\{\frac{C \nu(k)}{2 \pi} \int_{[-\pi, \pi]} \cos ^{2 k} \frac{t}{2} d t\right\} \cos ^{2 k}(\eta / 2)
$$


Thus, the de la Vallée Poussin kernel is invariant under the projection operator and provides a central (rotationally invariant) model odf and pdf, respectively. The order of approximation of the corresponding singular integral is rather poor (Butzer and Nessel, 1971); nevertheless, the de la Vallée Poussin kernel may provide an appropriate and computationally efficient model distribution.

\subsection{Abel-Poisson kernel - Cauchy distribution - "Lorentzian" standard distribution}

Applying the pole figure projection operator (1) to the Abel-Poisson kernel (cf. Butzer and Nessel, 1971) on $S^{4} \subset I R^{4}$

$$
\begin{aligned}
\mathcal{L}_{4}\left(\omega, \kappa^{2}\right)= & C_{L A}(\kappa) \sum_{l=0}^{\infty}(2 l+1) \kappa^{2 l} D_{l}(\omega) \\
=\left(1-\kappa^{2}\right) & \frac{\left(1+\kappa^{2}\right)^{2}+4 \kappa^{2} \cos ^{2}(\omega / 2)}{\left[\left(1+\kappa^{2}\right)^{2}-4 \kappa^{2} \cos ^{2}(\omega / 2)\right]^{2}} \\
& \omega \in[0, \pi], \kappa \in[0,1), \kappa_{0}=1-
\end{aligned}
$$

yields

$$
\begin{aligned}
P_{\mathrm{x}}\left[\mathcal{L}_{4}\left(\omega, \kappa^{2}\right)\right](\mathrm{y}) & =C_{\mathcal{L}_{3}}(\kappa) \sum_{l=0}^{\infty}(2 l+1) \kappa^{2 l} P_{l}\left(\mathrm{x} g_{0} \mathrm{y}\right) \\
& =\frac{1-\kappa^{4}}{\left(1+\kappa^{4}-2 \kappa^{2} \mathrm{x} g_{0} \mathrm{y}\right)^{3 / 2}}=\mathcal{L}_{3}\left(\eta, \kappa^{2}\right)
\end{aligned}
$$

the Abel-Poisson kernel (on $S^{3} \subset I R^{3}$ ). The result is contained in (Matthies et al., 1987) where the invariance was not noticed. The term "Lorentzian" standard distribution was coined, but above all it should be referred to as a multivariate generalization of the circular wrapped Cauchy distribution (cf. Mardia, 1972).

Summarily, the Abel-Poisson kernel is preserved by the projection operator and provides a central (rotationally invariant) model odf and pdf, respectively.

In 1- dimensional theory for a function defined on the circle, its corresponding singular integral is related to a Laplace differential equation and provides a solution of Dirichlet's problem for the unit disc, i.e. it is a harmonic function.

\subsection{Gauss-Weierstrass kernel - Brownian distribution - "normal" distribution}

Applying the pole figure projection operator (1) to the Gauss-Weierstrass kernel (cf. Butzer and Nessel, 1971)

yields

$$
\begin{gathered}
B_{4}(\omega, \kappa)=C_{B 4}(\kappa) \quad \sum_{l=0}^{\infty}(2 l+1) \exp [-l(l+1) \kappa] D_{l}(\omega) \\
\omega \in[0, \pi], \kappa \in(0,1], \kappa_{0}=0+
\end{gathered}
$$

$$
P_{\mathrm{x}}\left[B_{4}(\omega, \kappa)\right](\mathrm{y})=C_{B 3}(\kappa) \sum_{l=0}^{\infty}(2 l+1) \exp [-l(l+1) \kappa] P_{l}\left(\mathrm{x} g_{0} \mathrm{y}\right)=B_{3}(\eta ; \kappa)
$$


The result is due to (Savyolova, 1984; 1989; see also Bucharova and Savyolova, 1993) who refers to the Gauss-Weierstrass kernel as to the spherical normal distribution satisfying the spherical central limit theorem. However, a spherical central limit theorem thought of as straightforward spherical generalization of the central limit theorem for Euclidean spaces does not exist. The Brownian distribution may be interpreted as multivariate generalization of the circular wrapped normal distribution (Mardia, 1972).

The Gauss-Weierstrass kernel is preserved by the projection operator and provides a central (rotationally invariant) model odf and pdf, respectively. The distribution is infinitely divisible and satisfies the convolution semi-group property (cf. Nikolayev and Ullemeyer, these proceedings).

Its corresponding singular convolution integral applied to a function $f$ provides a solution of a diffusion problem with initial conditions given by $f$.

\section{COUNTEREXAMPLES}

\subsection{Fisher distribution on SO(3) - Bingham distribution on $S_{+}^{4} \subset I R^{4}$ - "Gaussian" standard distribution}

Applying the pole figure projection operator (1) to the Fisher matrix distribution on $S O(3)$ when it is central and reduces to the von Mises-Fisher distribution, or equivalently to the Bingham distribution on $S^{4}$ when it reduces to the rotationally invariant Watson distribution

yields

$$
\begin{gathered}
w_{4}(\omega ; \kappa)=C w_{4}(\kappa) \exp (\kappa \cos \omega)=C_{4}(\lambda) \exp \left(\lambda \cos ^{2} \frac{\omega}{2}\right) \\
\omega \in[0, \pi], \kappa, \lambda \in I R_{+}, \kappa_{0}=\lambda_{0}=\infty
\end{gathered}
$$

which is different from the von Mises-Fisher distribution on $S^{3}$

$$
\mathcal{W}_{3}(\eta, \kappa)=C_{3}(\kappa) \exp (\kappa \cos \eta)
$$

Thus, the von Mises-Fisher distribution is not preserved by the projection operator; nevertheless, it provides a useful central (rotationally invariant) model odf labeled Gaussian standard distribution in (Matthies et al., 1987). The distribution is infinitely divisible (Kent, 1977).

\subsection{Riemann-Lebesgue kernel, Steklov mean}

Applying the pole figure projection operator (1) to the Riemann-Lebesgue kernel (cf. Butzer and Nessel, 1971)

yields

$$
\begin{aligned}
I_{0}(\omega, \kappa)= & C_{I_{0}}(\kappa) 1_{((\kappa+1) / 2,1]}\left(\cos ^{2}(\omega / 2)\right) \\
= & C_{I_{0}}(\kappa) 1_{(\kappa, 1]}(\cos \omega) \\
& \omega \in[0, \pi], \kappa \in[-1,1), \kappa_{0}=1-
\end{aligned}
$$

$$
\mathrm{P}_{\mathrm{x}}\left[I_{0}(\omega, \kappa)\right](\mathrm{y})=\frac{4 C_{[0}(\kappa)}{2 \pi} \arccos \frac{\cos \omega_{b} / 2}{\cos \eta / 2} 1_{(\kappa, 1]}(\cos \eta)
$$

for $\eta \leq \omega_{\mathrm{b}}$ with $\omega_{\mathrm{b}}=\arccos \kappa$. 
The notationally obvious generalization with $k \in I N$ (which leads in 1-dimensional theory to the introduction of splines by iterated singular integrals)

$$
\begin{aligned}
g_{k}(\omega ; \kappa)= & C_{g_{k}}(\omega)\left(\frac{\cos ^{2}(\omega / 2)-(\kappa+1) / 2}{1-(\kappa+1) / 2}\right)^{k} 1_{((\kappa+1) / 2,1]}\left(\cos ^{2}(\omega / 2)\right) \\
= & C_{g_{k}}(\omega)\left(\frac{\cos \omega-\kappa}{1-\kappa}\right) 1_{(\kappa, 1]}(\cos \omega) \\
& \omega \in[0, \pi], \kappa \in[-1,1), \kappa_{0}=1-
\end{aligned}
$$

yields for $k=1$

$$
\begin{aligned}
P_{\mathrm{x}}\left[\mathcal{J}_{k}(\omega ; \kappa)\right](\mathrm{y}) & =\left\{\frac{2 C_{g_{k}}(\omega)}{2 \pi} \int_{\left[0, t_{b}\right]} \frac{2 \cos ^{2} \frac{\eta}{2} \cos ^{2} \frac{t}{2}-1-\kappa}{1-\kappa} d t\right\} 1_{(\kappa, 1]}(\cos \eta) \\
& =\frac{C_{g_{k}}(\omega)}{2 \pi} \frac{2}{1-\kappa}\left\{\left(t_{b}+\sin t_{b}\right) \cos ^{2} \frac{\eta}{2}-(1+\kappa) t_{b}\right\} 1_{(\kappa, 1]}(\cos \eta)
\end{aligned}
$$

for $\eta \leq \omega_{\mathrm{b}}$. Thus, the Riemann-Lebesgue kernel is neither invariant under the projection operator nor does it seem to possess any particularly appealing properties.

\subsection{Indicators with respect to partitions}

Let $\mathcal{G}(N)=\left\{G_{n} \mid n=1, \ldots, N\right\}$ be a partition of $G \subset S O(3)$, i.e. $\cup G_{n}=G, G_{n} \cap G_{m}$ $=\varnothing$ if $m \neq n$, and let

$$
1_{G n}(g)= \begin{cases}1 & \text { if } g \in G_{n} \in \mathcal{G}(N) \\ 0 & \text { otherwise }\end{cases}
$$

denote the indicator function with respect to $G_{n} \in \mathcal{G}(N)$. For every $N \in I N$ the set $\left\{1_{G n}, n=1, \ldots, N\right\}$ is an orthonormal total and closed system of functions defined on $G$.

The analogue is true for a partition $Z(P)=\left\{Z_{p} \mid p=1, \ldots, P\right\}$ of $S_{+}^{3}$ and corresponding indicator functions $1_{Z_{p}}(\mathrm{r})$ with respect to $\mathcal{L}^{2}\left(S_{+}^{3}\right)$

Let $\mathrm{x} \in Z_{p 2} \subset S_{+}^{3}$ be fixed; applying the projection $P_{\mathrm{x}}$ to $1_{G n_{0}}$ with $G_{n 0} \ni \mathrm{g}_{n 0}$ such that $g_{n_{0}} \mathrm{y}=\mathrm{x}$ for some $\mathrm{y} \in Z_{p 1} \subset S_{+}^{3}$ yields

$$
P_{\mathrm{X}}\left[1_{G n 0}(g)\right](\mathrm{y}) \neq \frac{1}{2 \pi} 1_{Z p 2}(\mathrm{x}) 1_{Z_{p 1}}(\mathrm{y})
$$

Thus, indicators with respect to given partitions are not preserved, i.e. canonical partitions of $G \subset S O(3)$ and $S^{3} \subset I R^{3}$ with respect to the pole figure projection operator do not seem to exist.

\section{METHODS IN TEXTURE GONIOMETRY}

\subsection{Quantitative Texture Analysis}

Let $\kappa_{4}(\omega ; \rho)$ be an even rotationally invariant approximate indentity on $S^{4}$ such that

$$
P_{\mathrm{x}}\left[\kappa_{4}\left(\omega\left(g_{0}^{-1} g\right) ; \rho_{4}\right)\right](\mathrm{y})=\kappa_{3}\left(\eta\left(\mathrm{x}, g_{0} \mathrm{y}\right) ; \rho_{3}\right)
$$


where $\kappa_{3}\left(\eta\left(\mathrm{x}, g_{0} \mathrm{y}\right) ; \rho_{3}\right)$ is the corresponding rotationally invariant approximate identity on $S^{3}$. Obviously, all the kernels shown are valid candidates.

An approximation of an orientation density function $f$ by the singular integral $A_{\rho}$ corresponding to the approximate identity $\kappa_{4}$ is then given by

$$
\left(A_{\rho}^{(4)} f\right)(g)=\sum_{n=1}^{N} f\left(g_{n}\right) \kappa_{4}\left(\omega\left(g g_{n}^{-1}\right) ; \rho_{4}\right)=\hat{f}(g)
$$

with $N \in I N$ large and a tuning parameter $\rho_{4}$ appropriately adjusted to the total number $N$ of sites $g_{n}, n=1, \ldots, N$. Next, the pole figure operator $\tilde{P}_{h}$ is applied to $\hat{f}$ which yields

$$
\left(\tilde{P}_{h} \hat{f}\right)(\mathrm{r})=\frac{1}{M_{h}} \sum_{n=1}^{N} f\left(g_{n}\right) \sum_{m=1}^{M_{h}} . \kappa_{3}\left(\mathrm{~h}_{m} g_{n} \mathrm{r} ; \rho_{3}\right)
$$

Finally, the unknown coefficients $f\left(g_{n}\right), n=1, \ldots, N$, of the expansion (27) are determined according to

$$
\frac{1}{M_{h}} \sum_{n=1}^{N} f\left(g_{n}\right) \sum_{m=1}^{M_{h}} \kappa_{3}\left(\mathrm{~h}_{m} g_{n} \mathrm{r} ; \rho_{3}\right)=\tilde{P}_{h}\left(\mathrm{r}_{l}\right)=\imath_{l}, l=1, \ldots, L
$$

with a large total number $L \in I N$ of measured intensities $\boldsymbol{l}_{l}$.

The system of linear equations (29) provides the general scheme of any particular advanced method of quantitative texture analysis except for the harmonic and indicator method. While the latter may be referred to as orthonormal series expansions (Schaeben, 1995b), the former may be characterized as nonorthogonal series expansion methods (cf. Freeden and Schreiner, 1994). The system is rank deficient, and additional mathematical modeling assumptions are required as usually to resolve the inverse problem of texture analysis in a unique way. Methods differ in the choice of modeling assumption(s), and algorithms for numerical determination of the corresponding model solution.

To be of some practical interest the kernels should be easy to evaluate numerically. Moreover, it should be noted that the matrix of the linear system corresponding to equations (28) and (29) is generally not sparse as for kernels with "local" support like $\delta$-distributions, indicators, Riemann-Lebesgue kernel.

\subsection{Exploratory Texture Modeling - Component Fit Methods}

As above, let $\kappa_{4}\left(\omega ; \rho_{4}\right)$ be an even (rotationally invariant) approximate identity on $S^{4}$ such that

$$
P_{\mathrm{x}}\left[\kappa_{4}\left(\omega\left(g_{0}^{-1} g\right) ; \rho_{4}\right)\right](\mathrm{y})=\kappa_{3}\left(\eta\left(\mathrm{x}, g_{0} \mathrm{y}\right) ; \rho_{3}\right)
$$

where $\kappa_{3}\left(\eta\left(\mathrm{x}, \mathrm{g}_{0} \mathrm{y}\right) ; \rho_{3}\right)$ is the corresponding rotationally invariant approximate identity on $S^{3}$. Let

$$
\hat{P}_{h}(\mathrm{r})=\frac{1}{M_{h}} \sum_{k=1}^{K} \sum_{m=1}^{M_{h}} \alpha_{k} \kappa_{3}\left(\mathrm{rr}_{k, m} ; \rho_{k}\right)
$$

be an approximation of $\tilde{P}_{h}$ by $K$ components $\left(K\right.$ small) each with parameters $\alpha_{k}, \mathrm{r}_{k, m}$, $\rho_{k}$ to be fit such that

$$
\hat{\tilde{P}}_{h}\left(\mathrm{r}_{l}\right) \approx \tilde{P}_{h}\left(\mathrm{r}_{l}\right)=\imath_{l}, l=1, \ldots, \mathrm{L}
$$


where $L$ denotes the total number of measured intensities $\iota_{l}$. Usually, this fit is basically done interactively by trial and error supported by computer graphics. It should be noted that for $M_{h}>2$ the geometry of the points $\mathrm{r}_{k, m} \in S_{+}^{3}, m=1, \ldots, M_{h} / 2$, for a given $k$ is imposed by the crystallographic symmetry of the crystal form $h$ and that for fixed $k$ the set $\left\{\mathrm{r}_{k, m}, m=1, \ldots, M_{h} / 2\right\}$ uniquely determines an orientation $g_{k} \in G$ such that $g_{k} \mathrm{r}_{k, m}=\mathrm{h}_{m}, m=1, \ldots, M_{h} / 2$.

Then

$$
\hat{f}(g)=\sum_{k=1}^{K} \alpha_{k} \kappa_{4}\left(\omega\left(g g_{k}^{-1}\right) ; \rho_{4}\right)
$$

provides an exploratory modeling of the inverse projection problem.

Any particular method of orientation component fit employs a scheme provided by equations (31) and (33) for a special choice of $\kappa_{4}$, and $\kappa_{3}$, respectively. Even though these methods differ in the extent of numerical sophistication, they all are nevertheless afflicted with the common problem that the number of components is obviously somewhat arbitrary and to some extent subjective. The only check is the goodness of fit of pole density functions, but that does not imply any measure of confidence or ghost correction capability with respect to the orientation density function determined in this way. Pole density component fit methods are much more apt to model a given experimental pole density function with a few evidently major components and to provide thus a better understanding of the pattern of preferred orientation (whatever the over all goodness of fit is) than to resolve the inverse problem of texture goniometry.

Comparing eq. (31) and eq. (29) reveals the fundamental difference between texture modeling by components and a full texture analysis with respect to a set of basis functions very clearly. Interpreting the superposition of few model functions as a particular finite series expansion, component fit methods seem applicable in terms of series expansion methods if only very few coefficients contribute considerably in the corresponding series expansion. If the complexity of the pattern of preferred crystallographic orientation requires a large number of rotationally invariant model functions for a sufficiently good fit, the method generalizes to a finite series expansion into non-orthogonal model functions.

While the kernels to be applied in nonorthogonal series expansion methods of texture analysis have to be rotationally invariant, application of model distributions without rotational symmetry seems favorable in exploratory texture modeling if the number of parameters to be fit remains reasonable small and if the parameters can actually be fit by an analysis of pole density functions (cf. Schaeben, 1990; 1995a; Eschner, 1993).

\section{APPLICATIONS IN ORIENTATION IMAGING MICROSCOPY}

Orientation imaging microscopy (Adams et al., 1992) does not provide mean intensities but direct measurements of localized orientations, i.e. of grains at specified locations

$$
g_{n}=g\left(\mathrm{u}_{n}\right)=1_{D k}\left(\mathrm{u}_{n}\right) g\left(D_{k}\right)
$$

with $\mathrm{u}_{n} \in D_{k} \subset I R^{3}, n=1, \ldots, N, k=1, \ldots, K$. Thus it provides the essential prerequisite of a complete orientation distribution analysis in Sander's sense of Achsenverteilungsanalyse (Sander, 1934; 1950) by spatial orientation correlation analysis. 


\subsection{Orientation Density Estimation by Orthogonal Series Expansion}

Harmonic series expansion yields an estimate of the orientation density by number emphasized by the superscript \#

$$
\begin{aligned}
\hat{f}^{\#}\left(g ; g_{1}, \ldots, g_{N}\right) & =\frac{1}{N} \sum_{i=1}^{N} f\left(g_{i}\right) \delta_{4}\left(g g_{i}^{-1}\right) \\
& =\sum_{l=0}^{\infty} \sum_{m=-1}^{l} \sum_{n=1}^{l} C_{l}^{m n} D_{m, n}^{l}\left(g^{-1}\right)
\end{aligned}
$$

with coefficients

$$
C_{l}^{m n}=(2 l+1) \frac{1}{N} \sum_{i=1}^{N} D_{m, n}^{* l}\left(g_{i}^{-1}\right)
$$

which is not generally nonnegative (cf. Kronmal and Tarter, 1968; Watson, 1969), and which requires a correction to be interpreted as an estimate of the common orientation density by volume (cf. Wright and Adams, 1990).

\subsection{Kernel Orientation Density Estimation}

The kernel estimate (Schaeben, 1982, 1994; Hall et al., 1987) of the probability density of orientations by number is defined as

$$
\hat{f}^{\#}\left(g, \rho ; g_{1}, \ldots, g_{N}\right)=\frac{1}{N} \sum_{n=1}^{N} \kappa_{4}\left(\omega\left(g g_{n}^{-1}\right) ; \rho_{4}(N)\right) \geq 0
$$

Let the sites $\mathrm{u}_{n} \in D \subset I R^{3}$ of orientation measurements $g_{n}=g\left(\mathrm{u}_{n}\right)$ be uniformly distributed in a domain $D$ and let $\left\{D_{k} \mid k=1, \ldots, K\right\}$ be a partition of $D$ into "grains". Provided the random variables $1_{D_{k}}\left(\mathrm{u}_{n}\right)$ and $g\left(D_{k}\right)$ are uncorrelated, then the kernel estimate of the probability density function of orientations by volume is defined as

$$
\begin{array}{r}
\hat{f}\left(g, \rho ; g\left(\mathrm{u}_{1}\right), \ldots, g\left(\mathrm{u}_{N}\right)\right)=\frac{1}{N} \sum_{n=1}^{N} \kappa_{4}\left(\omega\left(g g^{-1}\left(\mathrm{u}_{n}\right)\right) ; \rho_{4}(N)\right) \\
=\frac{1}{v(D)} \sum_{k=1}^{K} v\left(D_{k}\right) \kappa_{4}\left(\omega\left(g g^{-1}\left(D_{k}\right)\right) ; \rho_{4}(N)\right)
\end{array}
$$

where $v\left(D_{k}\right) / v(D)$ denotes the volume portion of grain $D_{k}$ within the domain $D$.

A corresponding pole density function can be calculated according to

$$
\tilde{P_{h}}[\hat{f}(g ; \rho)](\mathrm{r})=\frac{1}{v(D)} \sum_{m=1}^{M_{h}} \sum_{k=1}^{K} v\left(D_{k}\right) \kappa_{3}\left(\mathrm{~h}_{m} g\left(D_{k}\right) \mathrm{r} ; \rho_{3}(N)\right)
$$

\section{CONCLUSIONS}

Numerical determination and evaluation of an orientation density function either from diffraction experiments or individual orientation measurements requires its representation by a finite series expansion, i.e. by a series expansion which is genuinely finite or finite by truncation. This communication presents a collection of families of functions 
which are appropriate for series expansions of orientation distributions. To be of practical interest in texture analysis, it is emphasized that the family of functions is not required to be orthonormal and closed (as are harmonics and indicators) nor to be invariant with respect to the pole figure projection operator (as are harmonics and distributions of Brownian and Cauchy type). The results are instructively summarized in table 1 .

Table 1 Summary: Methods in QTA

\begin{tabular}{|c|c|c|c|}
\hline function & $\begin{array}{l}\text { invariant } \\
+ \text { yes; } ; \text { - no) }\end{array}$ & $\begin{array}{l}\text { orthonormal } \\
(+ \text { yes; }- \text { no) }\end{array}$ & related $\operatorname{method}(s)$ \\
\hline harmonics & + & + & harmonic method(s) \\
\hline Dirac $\delta$ & + & + & ideal orientations, discrete methods \\
\hline indicators & - & + & discrete methods \\
\hline Watson-Fisher "Gaussian" & - & - & $\begin{array}{l}\text { component fit, modeling, series } \\
\text { expansion, density estimation }\end{array}$ \\
\hline Brownian "normal" & + & - & $\begin{array}{l}\text { component fit, modeling, series } \\
\text { expansion, density estimation }\end{array}$ \\
\hline Cauchy "Lorentzian" & + & - & $\begin{array}{l}\text { component fit, modeling, series } \\
\text { expansion, density estimation }\end{array}$ \\
\hline
\end{tabular}

\section{References}

Adams, B. L., Wright, S. I. and Kunze, K. (1992). Orientation imaging - The emergence of a new microscopy: preprint, Metall. Trans., A.

Bucharova, T. I. and Savyolova, T. I. (1993). Application of normal distributions on $S O(3)$ and $S^{n}$ for orientation distribution function approximation: Textures and Microstructures, 21, 161-176.

Bunge, H. J. (1969). Mathematische Methoden der Texturanalyse, Akademie Verlag, Berlin.

Bunge, H. J. (trans. P. R. Morris) (1982). Texture Analysis in Materials Science, Butterworths, London.

Butzer, P. L. and Nessel, R. J. (1971). Fourier Analysis and Approximation: Birkhäuser Verlag, Basel and Stuttgart.

Eschner, T. (1993). Texture analysis by means of model functions: Textures and Microstrutures, 21, 139-146.

Freeden, W. and Schreiner, M. (1994). Nonorthogonal expansions on the sphere: MMAS, to appear.

Hall, P., Watson, G. S. and Cabrera, J. (1987). Kernel density estimation with spherical data: Biometrika, 74, 751-762.

Kent, J. T. (1977). The infinite divisibility of the von Mises-Fisher distribution for all values of the parameter in all dimensions: Proc. London Math. Soc., (3) 35, 359-384.

Kronmal, R. and Tarter, M. (1968). The estimation of probability densities and cumulatives by Fourier series methods: J. Amer. Stat. Assoc., 63, 925-952.

Mardia, K. V. (1972). Statistics of Directional Data: Academic Press, New York.

Matthies, S., Vinel, G. W. and Helming, K. (1987, 1988, 1990). Standard Distributions in Texture Analysis I, II, III, Akademie Verlag, Berlin.

Sander, B. (1934). Fortschritte der Gefügekunde der Gesteine, Anwendungen, Ergebnisse, Kritik: Fortschr. Mineral. Petrogr., 18, 111-170.

Sander, B. (1950). Einführung in die Gefügekunde der geologischen Körper, Vol 2: Springer, Vienna.

Savelova, T. I. (identical with Savyolova, T. I.), (1984). Distribution functions of grains with respect to orientation in polycrystals and their Gaussian approximation: Industrial Laboratory, 50, 468-474; translated from Zavodskaya Laboratoriya, 50, 48-52. 
Savelova, T. I. (identical with Savyolova, T. I.), (1989). Computing polar figures and deriving orientation distributions from them for canonical Gaussian distributions: Industrial Laboratory, 55, 1045-1048; translated from Zavodskaya Laboratoriya, 55, 57-60.

Schaeben, H. (1982). Fabric-diagram contour precision and size of counting element related to sample size by approximation theory methods: Math. Geol., 14, 205-216; Erratum: Math. Geol., 15, 579-580

Schaeben, H. (1990). Parameterizations and probability distributions of orientations: Textures and Microstructures, 13, 51-54.

Schaeben, H. (1994). Diskrete mathematische Methoden zur Berechnung und Interpretation von kristallographischen Orientierungsdichten: DGM Informationsgesellschaft, Oberursel.

Schaeben, H. (1995a). A note on a generalized standard orientation distribution in pdf-component fit methods: Textures and Microstructures, 23, 1-5.

Schaeben, H. (1995b). Analogy and duality of texture analysis by harmonics or indicators: J. Sci. Comp., to appear

Wright, S. I. and Adams, B. L. (1990). An evaluation of the single orientation method for texure determination in materials of moderate texture strength: Textures and Microstructures, 12, 65-76

Watson, G. S. (1969). Density estimation by orthogonal series: Ann. Math. Stat., 40, 1496-1498. 